\title{
ALMACÉN DE CARBONO EN BIOMASA AÉREA DE PLANTACIONES EXPERIMENTALES CON ESPECIES DE SUCESIÓN TEMPRANA DEL BOSQUE MESÓFILO DE MONTAÑA
}

\section{STORE CARBON IN ABOVEGROUND BIOMASS OF EXPERIMENTAL PLANTATIONS WITH SPECIES OF EARLY SUCCESSIONAL CLOUD FOREST}

\author{
María de la luz Avendaño-Yáñez ${ }^{1}$, Lázaro Rafael Sánchez-VelásqueZ², Daniela Martínez-Ramírez ${ }^{3}$, Yareni \\ Perroni², Sara Patricia IbarRa-Zavaleta², EnRique Alarcón ${ }^{2}$ y María del Rosario Pineda-López ${ }^{*}$
}

${ }^{1}$ INBIOTECA. Universidad Veracruzana, Xalapa, Veracruz, México. Actualmente Investigador independiente. ${ }^{2}$ INBIOTECA, Universidad Veracruzana, Xalapa, Veracruz, México.

${ }^{3}$ Instituto Tecnológico Superior de Jesús Carranza, Jesús Carranza, Veracruz

*Autor de correspondencia: rpineda@uv.mx

\section{Resumen}

Antecedentes: Las plantaciones forestales con especies nativas de crecimiento rápido contribuyen a la captura y almacenamiento de Carbono (C). Sin embargo, se sabe poco sobre la supervivencia, el potencial de captura y almacenamiento de $\mathrm{C}$ en plantaciones con especies nativas del bosque mesófilo de montaña (BMM).

Preguntas: ¿Es la supervivencia diferente entre Alnus acuminata y Trema micrantha sembrados en plantaciones?, ¿Son diferentes, la tasa anual de captura de carbono y el contenido de $\mathrm{C}$ total, entre ambas especies?

Especies de estudio: A. acuminata y T. micrantha, de estados sucesionales tempranos.

Sitio de estudio y fechas: Centro estado de Veracruz. 2013.

Método: En plantaciones de A. acuminata y T. micrantha de tres años y medio de edad, se registró la supervivencia, el diámetro y la altura. Se estimó el volumen, el área basal, el peso de la biomasa aérea, el contenido de $\mathrm{C}$ y las tasas anuales de crecimiento en altura, diámetro y contenido de carbono.

Resultados: La supervivencia de $A$. acuminata fue mayor que $T$. micrantha $(P<0.05)$. Las plantaciones de $A$. acuminata almacenaron más $\mathrm{C}\left(12.55 \pm 0.05 \mathrm{Mg} \mathrm{C}^{-1}\right)$ que las plantaciones de T. micrantha $\left(11.62 \pm 0.04 \mathrm{Mg} \mathrm{C} \mathrm{ha}^{-1}\right)(P<0.001)$. La tasa anual de captura de carbono en $A$. acuminata fue mayor $\left(2.6 \pm 0.0001 \mathrm{Mgha}^{-1} \mathrm{año}^{-1}\right)$ que T. micrantha $\left(2.02 \pm 0.00001 \mathrm{Mg} \mathrm{ha}^{-1} \mathrm{año}^{-1}\right)$.

Conclusiones: Las plantaciones con especies nativas de sucesión temprana representan una estrategia en acciones de restauración del BMM y constituyen sumideros de carbono que pueden contribuir a la mitigación del cambio climático.

Palabras clave: Alnus acuminata, bosque de niebla, restauración, Trema micrantha

\section{Abstract}

Background: Forest plantations with fast-growing native species contribute to capture and storage Carbon (C). However, little is known about the survival, potential of capture and storage of $\mathrm{C}$ in plantations with native species of mountain cloud forests (MCF) in Mexico.

Questions: Is the survival different between individuals of Alnus acuminata and Trema micrantha sown in experimental plantations? Is the annual carbon capture rate and the total $\mathrm{C}$ content between these two species different?

Species of study: A. acuminata and T. micrantha, species of rapid growth of the MCF.

Study site and years of study: Central area of the state of Veracruz. 2009-2012.

Method: A. acuminata and T. micrantha experimental plantations of three years an a half years old, survival, diameter and height were recorded. The volume, basal area, weight of aerial biomass, carbon content of trees and height, diameter and carbon growth rate were estimated.

Results: The survival of $A$. acuminata plants was greater than $T$. micrantha $(\mathrm{P}<0.05)$. The plantations of $A$. acuminata stored significantly more $\mathrm{C}\left(12.55 \pm 0.05 \mathrm{Mg} \mathrm{C} \mathrm{ha}^{-1}\right)$ than the plantations of $T$. micrantha $\left(11.62 \pm 0.042 \mathrm{Mg} \mathrm{C} \mathrm{ha}^{-1}\right)(P<0.001)$. The annual rate of carbon capture in A. acuminata was higher $\left(2.6 \pm 0.0001 \mathrm{Mg} \mathrm{ha}^{-1} \mathrm{yr}^{-1}\right)$ than T. micrantha $\left(2.02 \pm 0.00001 \mathrm{Mg} \mathrm{ha}^{-1} \mathrm{yr}^{-1}\right)(P<0.001)$.

Conclusions: Plantations with native species of early succession represent a complementary strategy in MCF restoration actions, as well as being carbon sinks that could contribute to mitigate climate change.

Keywords: Alnus acuminata, cloud forest, restoration, Trema micrantha

This is an open access article distributed under the terms of the Creative Commons Attribution License CCBY-NC (4.0) international. (c) (i) (8) https://creativecommons.org/licences/by-nc/4.0/ 
Los bosques tropicales almacenan $45 \%$ del carbono (C), por lo que constituyen el principal sumidero en ambientes terrestres (Bonan 2008, Beer et al. 2010). Los bosques mesofilos de montaña (BMM) o bosques de niebla (tropical montane cloud forests) capturan y almacenan importantes cantidades de C, aunque el papel de este tipo de bosques ha sido escasamente estudiado (Fehse et al. 2002, Spracklen \& Righelato 2014). La biomasa aérea ha sido el principal almacén de $C$ estudiado hasta ahora en los BMM (Spracklen \& Righelato 2016). A pesar de la importancia que este ecosistema tiene como sumidero de $\mathrm{C}$, actualmente presenta la tasa de deforestación más alta de los bosques tropicales (Lamb et al. 2005, Cayuela et al. 2006, Bruijnzeel et al. 2010), razón por la cual se han implementado estrategias para su restauración y conservación (Bubb et al. 2004, Avendaño-Yáñez et al. 2014). Acciones de restauración, como el establecimiento de plantaciones experimentales con especies autóctonas del BMM, representan también una opción para mitigar los efectos del cambio climático (Locatelli et al. 2015), por lo que estas plantaciones generan oportunidades para evaluar el almacén de carbono en la biomasa aérea de especies nativas poco utilizadas en programas de reforestación, pero con gran potencial para la restauración y el manejo forestal del BMM (Avendaño-Yáñez et al. 2014).

Uno de los aspectos principales en plantaciones de restauración y/o reforestación es la supervivencia y establecimiento de las plantas (Pareliussen et al. 2006, Birkinshaw et al. 2009). La supervivencia para algunas especies del BMM varían entre 2 y $94 \%$, dependiendo de las condiciones del hábitat (Ramírez-Bamonde et al. 2005, Muñiz-Castro et al. 2015). Sin embargo, hay poca información sobre la supervivencia de especies de rápido crecimiento y de estados sucesionales tempranos, además poco se sabe sobre la capacidad de captura y almacenamiento de C (por ej. Acosta 2003, Rodríguez-Laguna et al. 2006, Spracklen \& Righelato 2014).

Dentro de la flora del BMM, se han registrado una gran cantidad de especies arbóreas de sucesión temprana y tardía (Sánchez-Velásquez et al. 2008), las cuales pueden asimilar y acumular diferencialmente el $\mathrm{CO}_{2}$ a través del tiempo. Alnus acuminata Kunth y Trema micrantha (L.) Blume, son especies de sucesión temprana y crecimiento rápido (Russo 1990, Condit et al. 1993, Murcia 1997, Vázquez-Yanes 1998, Velázquez \& Gómez-Sal 2009) asociadas a la vegetación secundaria del BMM. Se sabe que las especies de crecimiento rápido pueden contribuir significativamente a la captura de carbono a partir de la producción de biomasa aérea y subterránea, en períodos relativamente cortos (De Deyn et al. 2008). Además, A. acuminata y T. micrantha, en particular tienen un gran potencial para facilitar el establecimiento de especies de sucesión intermedia y tardía con fines de restauración del BMM (Avendaño-Yáñez et al. 2014).

Si bien las especies de crecimiento rápido del BMM pueden contribuir de manera sustancial en la captura y el almacén de C (Shimamoto et al. 2014), es necesario primero, conocer la supervivencia de individuos en plantaciones con especies nativas y en segundo lugar conocer su capacidad de almacenamiento de $\mathrm{C}$ a través del tiempo. Las preguntas de este estudio fueron: 1) ¿Es la supervivencia diferente entre individuos de Alnus acuminata y Trema micrantha sembrados en plantaciones experimentales?, 2) ¿Son diferentes, la tasa anual de captura de carbono y el contenido de $\mathrm{C}$ total, entre ambas especies? Dado que ambas especies son de crecimiento rápido se espera que tengan una supervivencia, captura de carbono y contenido de $\mathrm{C}$ total similares.

\section{Materiales y métodos}

Área de estudio. El estudio se realizó en cuatro localidades de la zona montañosa central del estado de Veracruz, México, dentro de un gradiente altitudinal de 1,000 a 1,800 m. El clima que predomina en la región es húmedo con tres estaciones marcadas a lo largo del año (secas, lluvias y "nortes"-eventos monzónicos de entrada de agua al continente-). Las lluvias ocurren durante el verano y en invierno, la humedad ocurre en forma de neblina. El rango de precipitación en la región es de 1,500 a $1,700 \mathrm{~mm}$ y la temperatura promedio anual es de $16^{\circ} \mathrm{C}$. Los suelos son principalmente de tipo Andosol y el tipo de vegetación corresponde a BMM (Rzedowski 2006).

Especies estudiadas. Se seleccionaron dos especies nativas de sucesión temprana del BMM, Alnus acuminata Kunth (Betulaceae), la cual es una especie semi-decidua (Russo 1990) que tiene una distribución altitudinal amplia que va de los 1,000 a los 2,500 m s.n.m. o más (González-Espinosa et al. 2011), y Trema micrantha (L.) Blume (Cannabaceae), especie perennifolia con una distribución altitudinal que va de los 0 a los 1,700 m s.n.m. (Vázquez-Yanes 1998, Velázquez \& Gómez-Sal 2009). Alnus acuminata y T. micrantha son favorecidas por disturbios naturales o antropogénicos y colonizan exitosamente áreas abiertas, alcanzando alturas de hasta 30 y $20 \mathrm{~m}$, respectivamente (González-Espinosa et al. 2011) (Figura 1).

Establecimiento de plantaciones y registro de variables. En el verano del 2009 se establecieron dos experimentos con plantaciones monoespecíficas de $A$. acuminata y $T$. micrantha, con dos réplicas por especie. Las semillas se recolectaron de árboles maduros y sanos (al menos diez de cada especie) en bosques de la misma región; cada semilla se colocó en bolsas de plástico $(15 \times 25 \mathrm{~cm}$ llenas de tierra del bosque $)$ y se mantuvieron en un vivero rústico donde permanecieron hasta que las plántulas fueron transportados al campo. Las semillas colectadas estuvieron disponibles en campo con una diferencia de tres meses, así que las plántulas de Trema micrantha estuvieron en el invernadero tres meses y para Alnus acuminata seis meses; las plántulas se mantuvieron siempre con riego constante cuando así se requería. Las plantas de ambas especies se establecieron en parcelas de $35 \times 35 \mathrm{~m}$ $\mathrm{y}$ en cada una se sembraron (de manera equidistante "tres bolillos") 280 plantas de Alnus acuminata y 280 plantas de Trema micrantha, con una densidad inicial de siembra de 2,500 árboles ha-1. La supervivencia (número de individuos) se registró después de 3.5 años, considerando el número inicial de individuos $(\mathrm{n}=280)$ menos los individuos muertos después de 3.5 años. La talla inicial promedio de las plantas de $A$. acuminata fue $64 \pm 26 \mathrm{~cm}$ de altura y $6.9 \pm 3.8 \mathrm{~mm}$ de 


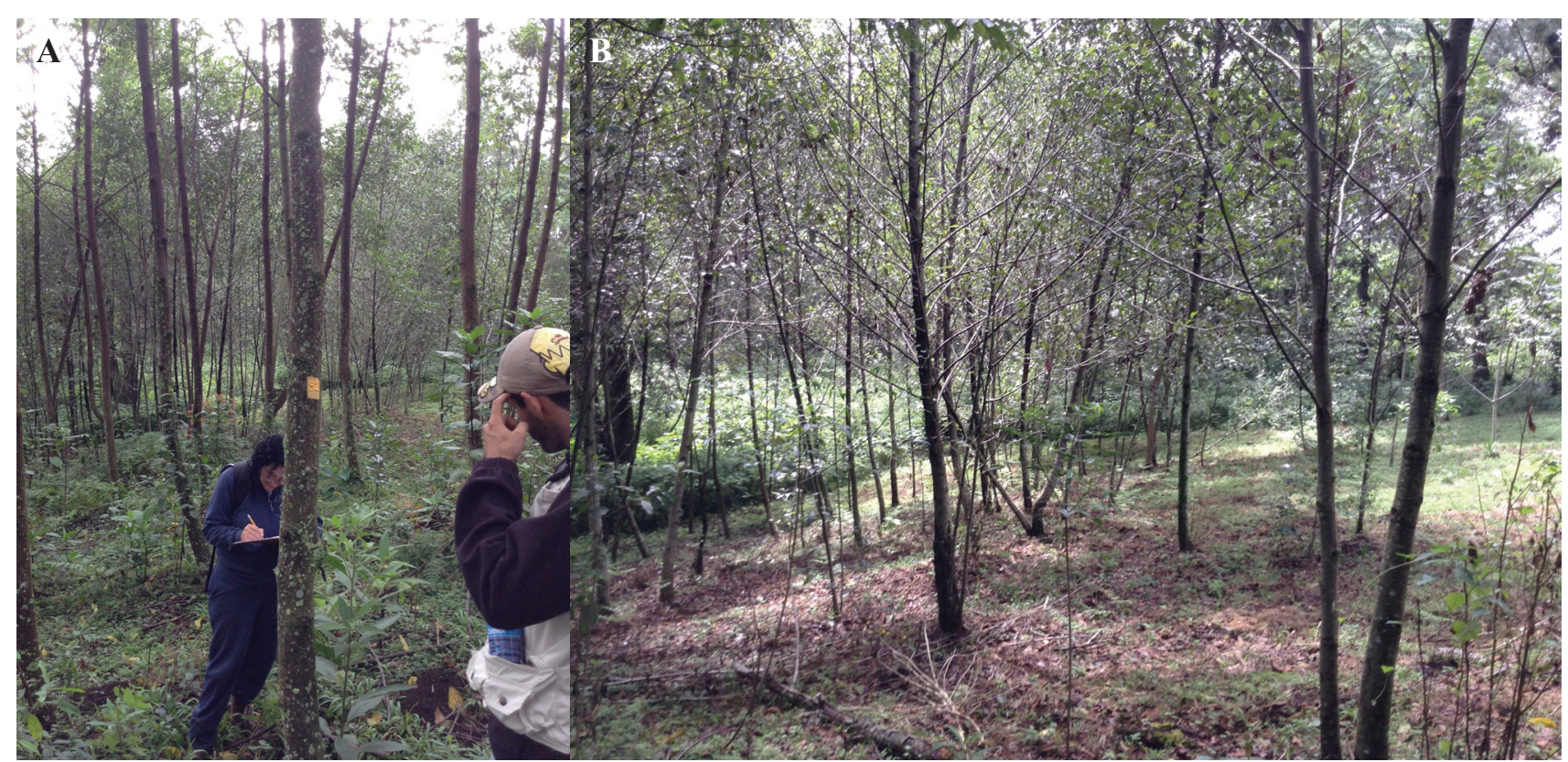

Figura 1. A. Plantaciones de 3.5 años de Trema micrantha, B. Alnus acuminata en el centro de Veracruz.

diámetro basal, mientras que para $T$. micrantha las medidas iniciales promedio fueron $25 \pm 7.6 \mathrm{~cm}$ de altura y $5.1 \pm 1.8$ mm de diámetro basal. Después de 3.5 años, estas mismas variables fueron registradas para ser usadas en la estimación de las tasas anuales de captura de $\mathrm{C}$, de crecimiento en altura y diámetro (ver más adelante). Cabe mencionar que el establecimiento de las plantaciones fue un experimento con fines de estudios sobre ecología de la restauración, por lo que un año después, bajo el dosel de $A$. acuminata y $T$. micrantha, se sembraron plantas de Juglans pyriformis Liebm., Quercus insignis Martens \& Galeotti y Oreomunnea mexicana (Stand1.) J.-F. Leroy subsp. mexicana, las cuales son especies de sucesión intermedia y avanzada. En estas últimas especies de crecimiento lento no se evaluó el contenido de carbono, debido a que eran muy pequeñas (dos años).

Estimación de la biomasa, almacén y tasa anual de captura de C. A partir de las variables de crecimiento descritas arriba (altura y diámetro), tanto al inicio como al final del experimento (después de 3.5 años), se estimaron otros atributos para cada una de las especies, a través de las siguientes ecuaciones:

Volumen del fuste:

$$
V=A B \times H \times F,
$$

Ec. 1

donde: $V$ es el volumen $\left(\mathrm{m}^{3}\right), A B$ es el área basal $\left(\mathrm{m}^{2}\right), H$ es la altura (m) y $F$ es el factor de corrección o coeficiente mórfico, una constante con valor de 0.7 (Dávalos-Sotelo 2008).

Área basal:

$$
A B=\pi / 4 \times d^{2},
$$

donde: d es el diámetro normal (m).
Peso de la biomasa aérea:

$$
W B A=V \times D A \times F E B,
$$

Ec. 3

donde: $W B A$ es el peso de la biomasa aérea (ton), $V$ es volumen, y $D A$ es densidad aparente de la madera $\left(\mathrm{t} \mathrm{m}^{-3}\right)$. La $D A$ para $A$. cuminata y $T$. micrantha fue de 0.40 y 0.42 , respectivamente. $F E B$ es factor de expansión de la biomasa, equivalente a 2.1 (Dávalos-Sotelo 2008).

Cantidad de carbono almacenado:

$$
C=W B A \times f C,
$$

Ec. 4

donde: $C$ es la cantidad de carbono almacenado $\left(\mathrm{Mg} \mathrm{C} \mathrm{ha}^{-1}\right)$, $W B A$ es biomasa $\left(\mathrm{Mg} \mathrm{C} \mathrm{ha}{ }^{-1}\right)$ y $f C$ es fracción estimada de carbono en la biomasa con un valor de 0.46 para $A$. cuminata y 0.4435 para $T$. micrantha (Pompa-García et al. 2017).

Cada valor de contenido de carbono por especie fue dividido por la superficie de los sitios de muestreo $\left(\mathrm{m}^{2}\right)$ para obtener $\mathrm{Mg} \mathrm{C} \mathrm{m}{ }^{-2}$ y después por hectárea ( $\mathrm{T} \mathrm{Mg} \mathrm{C} \mathrm{ha-1)}$ (Márquez 2000). A partir de esto se obtuvo el contenido de $\mathrm{C}$ para la biomasa aérea en las plantaciones de $A$. acuminata y $T$. micrantha expresado en $\mathrm{Mg} \mathrm{C} \mathrm{ha}^{-1}$.

Tasas anuales de captura de carbono, altura y diámetro:

$$
T A C C=(\ln C C 2-\ln C C 1) / t, \quad \text { Ec. } 5
$$

donde: $T A C C$ es la tasa anual de contenido de carbono, ln logaritmo natural, $C C 2$ es el contenido de carbono al final del experimento, $C C 1$ es el contenido de carbono al inicio del experimento y $t$ es el tiempo en años, en este caso $t=3.5$. Con esta misma ecuación se estimaron las tasas de crecimiento para la altura y el diámetro, es decir se sustituyeron en la Ec. 
5 las $C C_{i}$, por la altura y el diámetro iniciales y finales. De esta manera, todas las tasas son independiente al contenido de carbono, a las alturas y los diámetros iniciales.

Análisis de datos. Se comparó la supervivencia de las plantas de A. acuminata y $T$. micrantha en las plantaciones experimentales con una prueba de $\chi^{2}$. Debido a que se cuenta con 2 réplicas por especie (dos parcelas de 1,225 $\mathrm{m}^{2}$ cada una), las comparaciones de las tasas de crecimiento en altura y diámetro, del contenido de $\mathrm{C}$ y la tasa anual de contenido de carbono estimados para $A$. acuminata vs $T$. micrantha, se realizaron a través de 1,000 iteraciones. En cada iteración, de cada una de las variables, se seleccionaron al azar 100 individuos de cada especie, utilizando el método Bootstrap sin reemplazo (Manly 2001). Para cada iteración y variable, se proyectaron a hectárea (con excepción de la altura y el diámetro, los cuales la unidad de comparación fue entre individuos). Los promedios de las variables se compararon entre especies con una prueba de $t$ de Student utilizando R (R Core Team 2013).

\section{Resultados}

Después de tres años y medio, la supervivencia de $A$. acuminata en las plantaciones experimentales fue significativamente mayor que la de $T$. micrantha $(77$ y $66 \%$, respectivamente; $\chi^{2}=4.4, P=0.03$ ); es decir, $A$. acuminata tuvo una densidad de aproximadamente 1,715 árboles ha ${ }^{-1}$, mayor que la de $T$. micrantha de 1,590 árboles ha-1 . La talla promedio fue $11.1 \pm 7.8 \mathrm{~m}$ de altura y $8.7 \pm 3.4 \mathrm{~cm}$ de diámetro normal (altura de medición a $1.3 \mathrm{~m}$ ) para Alnus acuminata, y una altura de $8.8 \pm 2.4 \mathrm{~m}$ y el diámetro normal de $9.2 \pm 5.1 \mathrm{~cm}$ para Trema micrantha; en ambos casos fueron significativamente diferentes entre especies $(t=474, P<0001$ y $t=212$ $P<0.001$, respectivamente). Las tasas de crecimiento fueron también significativamente diferentes entre $T$. micrantha $\mathrm{y}$ A. acuminata. La altura fue mayor en A. acuminata (1.52 \pm 0.0004 vs $0.92 \pm 0.0003 ; t=433, P<0.0001)$ y en el diámetro normal fue $T$. micrantha $(0.81 \pm 0.0003$ vs $0.679 \pm$ 0.0005; $t=212, P<0.0001)$.

El contenido de $\mathrm{C}$ total estimado en la biomasa aérea fue significativamente mayor $(t=13.99, P<0.0001)$ en las plantaciones de $A$. acuminata $\left(12.55 \pm 0.05 \mathrm{Mg} \mathrm{C} \mathrm{ha}^{-1}\right) \mathrm{vs}$ T. micrantha $\left(11.62 \pm 0.04 \mathrm{Mg} \mathrm{C} \mathrm{ha}^{-1}\right)$ (Figura 2). La tasa anual de captura de carbono en $A$. acuminata fue mayor (2.6 $\left.\pm 0.0001 \mathrm{Mgha}^{-1} \mathrm{año}^{-1}\right)$ que T. micrantha $(2.02 \pm 0.00001$ $\mathrm{Mgha}^{-1}$ año $\left.^{-1}\right)(t=13.99, P<0.001)$.

\section{Discusión}

Después de 3.5 años, la densidad de arboles en las plantacionesde $A$. acuminata vs $T$. micrantha, fueron significativamente diferentes $(P<0.001)$, debido a una mayor supervivencia de individuos de $A$. acuminata. Esto tiene implicaciones en la acumulación de biomasa y $\mathrm{C}$, dado que la densidad de individuos en las plantaciones incide en las tasas de crecimiento de la biomasa. Sin embargo, es necesario detectar la densidad óptima de individuos para cada especie y sitio, porque muy

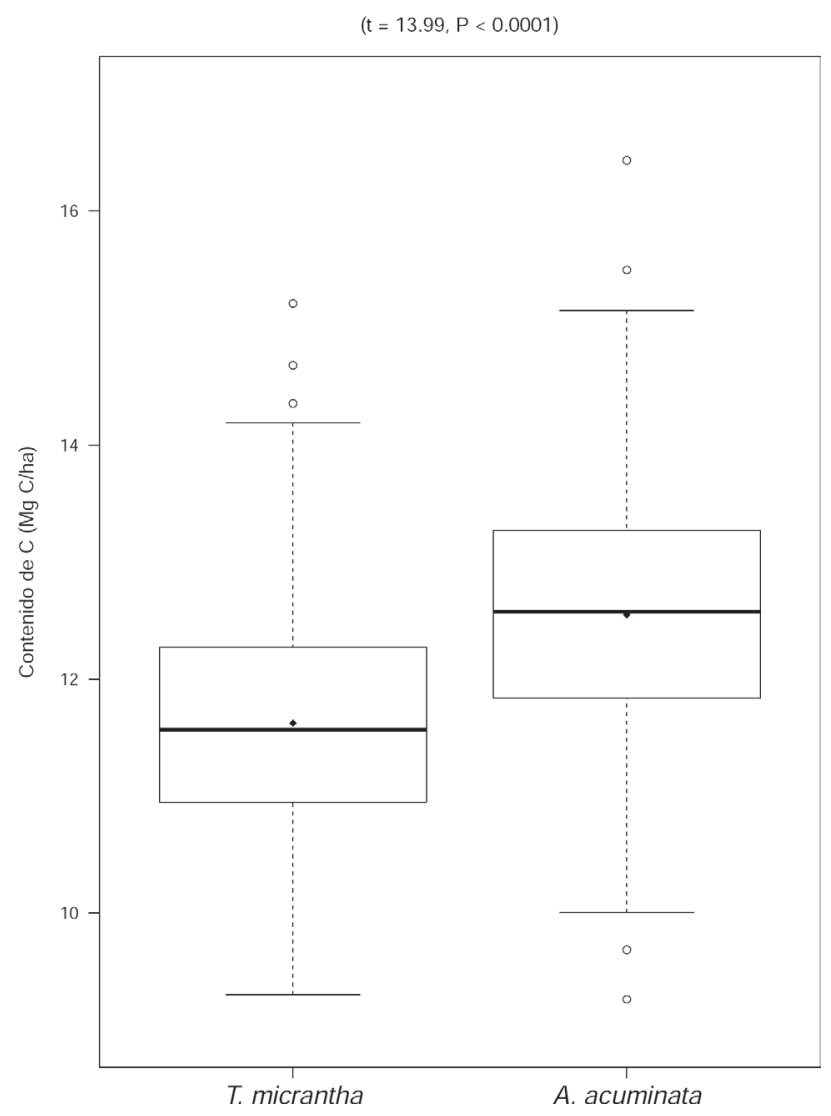

Figura 2. Comparación del contenido promedio por hectárea de $\mathrm{C}$ en la biomasa aérea entre Alnus acuminata y Trema micrantha. Resutados después de tres años y medio de crecimiento, en plantaciones experimentales de la región central montañosa del estado de Veracruz, México. Las diferencias entre especies fueron significativas ( $t=13.99$, $P<0.0001)$.

bajas densidades de siembra suelen ser poco eficientes en la captura de C (Swamy \& Mishra 2014). Una densidad adecuada, junto con el incremento de la edad de la plantación dan como resultado una mayor acumulación de $\mathrm{C}$ en la biomasa aérea (Yoda 1963, Du et al., 2015).

La tasa de crecimiento en altura y diámetro de las plantas implica una estrecha relación con el incremento de biomasa y el almacén de C. A. acuminata crece verticalmente con una tasa de crecimiento anual en altura de $1.52 \pm 0.0004 \mathrm{~m}$ (vs. $0.92 \pm 0.0003 \mathrm{~m}$ de $T$. micrantha), T. micrantha tiene una tasa de crecimiento anual en dap de $0.81 \pm 0.0003 \mathrm{~cm}$ (vs. $0.679 \pm 0.0005 \mathrm{~cm}$ de $A$. acuminata). Las tasas anuales del diámetro son menores que las encontradas por MendozaHernández (2015); dap $1.48 \mathrm{~cm}$ y $0.95 \mathrm{~cm}$. para A. acuminata y $T$. micrantha, respectivamente. Sin embargo, estos últimos resultados fueron obtenidos solo del último año de crecimiento de arboles de entre 30 y $40 \mathrm{~cm}$ de diámetro. Esto sugiere que la tasa de crecimiento del dap que registramos en este trabajo podría continuar aumentado con el tiempo, pero esto deberá ser monitoreado para llegar a probar esta hipótesis. 
Avendaño-Yáñez et al. / Botanical Sciences 97 (1): 82-88. 2019

Tabla 1. Algunos atributos funcionales de Alnus acuminata y Trema micrantha, dos especies de sucesión temprana utilizadas en las plantaciones experimentales. AFE área foliar específica, CE Conductancia estomática, DM Densidad de la madera.

\begin{tabular}{lll}
\hline Características & Alnus acuminata (Kunth.) & Trema micrantha $(\mathbf{L})$. \\
\hline Fenología foliar & Semi-decidua (1) & Perennifolia (2) \\
AFE & $67.38 \mathrm{~cm}^{2} \mathrm{~g}^{-1}(3)$ & $630.8 \mathrm{~cm}^{2} \mathrm{~g}^{-1}(4)$ \\
CE & $0.433 \mathrm{~mol} \mathrm{~m}^{-2} \mathrm{~s}^{-1}(5)$ & $0.539 \mathrm{~mol} \mathrm{~m}^{-2} \mathrm{~s}^{-1}(6)$ \\
DM & $0.40 \mathrm{~g} \mathrm{~cm}^{-3}(7)$ & $0.42 \mathrm{~g} \mathrm{~cm}^{-3}(8)$ \\
Fijación de N & Simbiosis con bacterias del género Frankia (9) & No se sabe \\
Micorrizas & Ectomicorrizas y micorrizas arbusculares (9 y 10) & Micorrizas arbusculares (11) \\
\hline
\end{tabular}

1) Russo 1990; 2) Vázquez-Yanes 1998; 3) Esperón-Rodríguez y Barradas 2015; 4) Datos no publicados de Guadalupe Hernández Vargas; 5) Esperón-Rodríguez y Barradas 2016; 6) Santiago y Wright 2007; 7) Bárcenas-Pazos y Dávalos-Sotelo 2001; 8) Bárcenas-Pazos y OrdoñezCandelaria 2008; 9) Russo 2005; 10) Becerra et al. 2005; 11)Siqueira y Saggin 2001.

Los resultados de este estudio también muestran que $A$. acuminata alcanza una tasa anual promedio de captura de $\mathrm{C}$ de $2.6 \pm 0.0001 \mathrm{Mg} \mathrm{Cha}^{-1} \mathrm{año}^{-1}$, y T. micrantha de $2.02 \pm$ $0.00001 \mathrm{Mg} \mathrm{Cha}^{-1} \mathrm{año}^{-1}$ en la biomasa aérea. Estos valores estimados son menores en comparación con otras especies de crecimiento rápido ampliamente seleccionadas por estos atributos, tales como Eucalyptus tereticornis $\mathrm{Sm}$. $\left(6 \mathrm{Mg} \mathrm{C}^{-1}\right.$ año ${ }^{-1}$ ) o Populus deltoides Marsh (8 $\mathrm{Mg} \mathrm{C} \mathrm{ha}^{-1}$ año $^{-1}$ ) (Kaul et al. 2010). Sin embargo, se esperaría que los valores de captura de $\mathrm{C}$ para $A$. acuminata y $T$. micrantha se incrementen con la edad de la plantación, como lo sugiere el estudio de Du et al. (2015) para plantaciones de Eucalyptus urophylla S. T. Blake y E. grandis W. Hill ex Maiden, con densidades intermedias que van de los 1,100 a 1,355 árboles ha ${ }^{-1}$. Swamy \& Mishra (2014) reportaron igualmente el incremento del C en plantaciones de Populus deltoides W. Bartram ex Marshal a mayor edad, aunque con densidades relativamente bajas (300, 400 y 625 árboles ha $\left.{ }^{-1}\right)$.

El C total capturado en la biomasa aérea de las plantaciones de A. acuminata $\left(12.55 \pm 0.05 \mathrm{Mg} \mathrm{C}^{-1}\right)$ y T. micrantha $\left.\left(11.62 \pm 0.04 \mathrm{Mg} \mathrm{C}^{-1}\right)^{-1}\right)$ fue significativamente diferente entre especies. Algunos estudios para el BMM reportan valores mayores, por ejemplo, Rodríguez-Laguna et al. 2006 reportaron $56.7 \mathrm{Mg} \mathrm{C}$ ha $^{-1}$ almacenado en los fustes de árboles de diferentes especies en un bosque maduro. Mientras que Acosta (2003) reportó, para el estrato arbóreo en dos $\mathrm{BMM}$, valores de 93.2 $\mathrm{Mg} \mathrm{C} \mathrm{ha}^{-1}$ en la región Mazateca y $30.5 \mathrm{Mg}$ C ha para la región Cuicateca, en la Sierra norte de Oaxaca. De acuerdo con los crecimientos que se obtuvieron en este estudio, la proyección para alcanzar $50 \mathrm{Mg} \mathrm{C} \mathrm{ha}^{-1}$, se lograría al rededor de los 12 años, tanto para $A$. acuminata como para $T$. micrantha. Si consideramos que los bosques intermedios y maduros del BMM ocurren después de los 50 años de un disturbio (Sánchez-Velásquez et al. 2008), entonces se observa el potencial de $A$. acuminata y $T$. micrantha para la captura de C.

A pesar de que Trema micrantha posee algunas características funcionales que pudieran conferirle ciertas ventajas de crecimiento y captura de $\mathrm{C}$, tales como el AFE y por ser una especie perenne (Tabla 1), solo se reflejó en el crecimiento diamétrico. Se requieren otros estudios para conocer la re- lación entre los atributos funcionales de estas especies y la captura de $\mathrm{C}$ incluyendo la parte subterránea.

En resumen, la reconversión de áreas deforestadas a bosques nativos mediante prácticas de restauración sucesional puede coadyuvar a la recuperación de reservorios o sumideros de carbono (Ciccarese et al. 2012). Las especies de sucesión temprana y crecimiento rápido, como $A$. acuminata y $T$. micrantha establecidas en plantaciones experimentales dentro de su rango de distribución natural en el BMM, representan una buena opción para la captura y almacenamiento de $\mathrm{CO}_{2}$ e inciar un proceso de restauración.

\section{Agradecimientos}

Agradecemos a los propietarios de los predios donde se establecieron las plantaciones, Raquel Cervantes, Álvaro Soberanes, Silvano Cortina y Jonás Tepatlán, así como a Rogelio Lara por su apoyo en campo. Este estudio fue financiado por el Fondo Sectorial de investigación para la educación SEP-CONACYT (CB-2010-0-156053). Se agradece a dos árbitros anónimos y al editor de sección por las sugerencias y observaciones, las cuales mejoraron el manuscrito final.

\section{Literatura citada}

Acosta MM. 2003. Diseño y aplicación de un método para medir los almacenes de carbono en sistemas con vegetación forestal y agrícolas de ladera en México. PhD Thesis. Colegio de Postgraduados.

Avendaño-Yáñez ML, Sánchez-VelásquezLR, Meave JA, Pineda-López MR. 2014. Is facilitation a promising alternative for cloud forest restoration? Forest Ecology and Management 329: 328-333.

DOI: http://doi.org/10.1016/j.foreco.2014.01.051

Bárcenas-Pazos G, Davalos-Sotelo R. 2001. Shrinkage values for 106 Mexican Woods. Journal of Tropical Forest Products 7(2): 125-134.

Bárcenas-Pazos G, Ordoñez-Candelaria VR. 2008. Calidad de la madera de los árboles de sombra. In: Manson RH, Hernández-Ortiz V, Gallina S, Mehltreter K, eds. Agroecosistemas cafetaleros de Veracruz: Biodiversidad, manejo y conser- 
vación. Instituto de Ecología A.C. e Instituto Nacional de Ecología, 235-246. ISBN: 970-709-112-6.

Becerra A, Zak MR, Horton TR, Micolini J. 2005. Ectomycorrhizal and arbuscular mycorrhizal colonization of Alnus acuminata from Calilegua National Park (Argentina). Mycorrhiza 15: 525-531.

DOI: http://doi.org/10.1007/s00572-005-0360-7

Beer C, Reichstein M, Tomelleri E, Ciais P, Jung M, Carvalhais N, Rödenbeck C, Arain A, Baldocchi D, Bonan GB, Bondeau A, Cescatti A, Lasslop G, Linroth A, Lomas M, Luyssaert S, Margolis H, Oleson KW, Roupsard O, Veenendaal E, Viovy N, Williams C, Woodward FI, Papale D. 2010. Terrestrial gross carbon dioxide uptake: global distribution and covariation with climate. Science 329: 834-838.

DOI: http://doi.org/10.1126/science.1184984.

Bonan GB. 2008. Forests and climate change: forcing, feedbac$\mathrm{ks}$, and the climate benefits of forests. Science 320: 14441449. DOI: http://doi.org/10.1126/science.1155121.

Birkinshaw C, Andrianjafy M, Rasolofonirina JJ. 2009. Survival and growth of seedlings of 19 native tree and shrub species planted in degraded forest as part of a forest restoration project in Madagascar's highlands. Madagascar Conservation and Development 4: 128-131.

Bruijnzeel LA, Kappelle M, Mulligan M, Scatena FN. 2010. Tropical montane cloud forests: state of knowledge and sustainability perspectives in a changing world. In: Bruijnzeel LA, Scatena FN, Hamilton L, eds. Tropical Montane Cloud Forests: Science for Conservation and Management. Cambridge University Press, 691-740. ISBN: 978-0-521-76035-5.

Bubb P, May I, Miles L, Sayer J. 2004. Cloud Forest Agenda. United Nations Environment Programme World Conservation Monitoring Centre, Cambridge. ISBN: 92-807-2399-5

Cayuela L, Golicher DJ, Benayas JMR, González-Espinosa M, Ramírez-Marcial N. 2006. Fragmentation, disturbance and tree diversity conservation in tropical montane forests. Journal of Applied Ecology 43: 1172-1181.

DOI: http://doi.org/10.1111/j.1365-2664.2006.01217.x.

Ciccarese L, Mattsson A, Pettenella D. 2012. Ecosystem services from forest restoration: thinking ahead. New Forests 43 : 543-560. DOI: http://doi.org/10.1007/s11056-012-9350-8.

Condit R, Hubbell SP, Foster RB. 1993. Identifying fast-growing native trees from the Neotropics using data from a large, permanent census plot. Forest Ecology and Management 62: 123-143. DOI: http://doi.org/10.1016/0378-1127(93)90046-P.

Dávalos-Sotelo R, Morato MI, Martínez Pinillos-Cueto E. 2008. Almacenamiento de carbono. In: Manson RH, HernándezOrtiz V, Gallina S, Mehltreter K, eds. Agroecosistemas cafetaleros de Veracruz: Biodiversidad, manejo y conservación. Instituto de Ecología A.C. e Instituto Nacional de Ecología, 223-234. ISBN: 970-709-112-6.

De Deyn G, Cornelissen J, Bardgett R. 2008. Plant functional traits and soil carbon sequestration in contrasting biomes. Ecology Letters 11: 516-531. DOI: http://doi.org/10.1111/j.1461-0248.2008.01164.x.

Du H, Zeng F, Peng W, Wang K, Zhang H, Liu L, Song T. 2015. Carbon Storage in a Eucalyptus Plantation chronosequence in Southern China. Forests 6: 1763-78. DOI: http://doi.org/10.3390/f6061763.
Esperón-Rodríguez M, Barradas VL. 2015. Ecophysiological vulnerability to climate change: water stress responses in four tree species from the central mountain region of Veracruz, Mexico. Regional Environmental Change 15: 93-108. DOI: http://doi.org/10.1007/s10113-014-0624-x.

Esperón-Rodriguez M, Barradas VL. 2016. Stomatal responses of tree species from the cloud forest in central Veracruz, Mexico. Botanical Sciences 94: 311-321. DOI: http://doi.org/10.17129/botsci.490.

Fehse J, Hofstede R, Aguirre A, Paladines C, Kooijman A, Sevink J. 2002. High altitude tropical secondary forests: a competitive carbon sink? Forest Ecology and Management 163: 9-25. DOI: http://doi.org/10.1016/S0378-1127(01)00535-7.

González-Espinosa M, Meave JA, Lorea-Hernández FG, IbarraManríquez G, Newton AC. 2011. The Red List of Mexican Cloud Forest Trees. Cambridge: Fauna \& Flora International. ISBN: 9781903703281.

Kaul M, Mohren GMJ, Dadhwal VK. 2010. Carbon storage and sequestration potential of selected tree species in India. Mitigation and Adaptation Strategies for Global Change 15: 489-510. DOI: http://doi.org/10.1007/s11027-010-9230-5.

Lamb D, Erskine PD, Parrota JA. 2005. Restoration of degraded tropical forests landscapes. Science 310: 1628-1632. DOI: http://doi.org/10.1126/science.1111773.

Locatelli B, Catterall CP, Imbach P, Kumar C, Lasco R, Marín-Spiotta E, Mercer B, Powers JS, Schwartz N, Uriarte M. 2015. Tropical reforestation and climate change: beyond carbon. Restoration Ecology 23: 337-34.

DOI: http://doi.org/10.1111/rec.12209.

Manly BFJ. 2001. Randomization, Bootstrap and Montecarlo Methods in Biology. Florida: Chapman \& Hall/CRC. ISBN: 1-58488-541-6.

Márquez L. 2000. Elementos técnicos para inventarios de carbono en uso de suelo. Fundación solar. Guatemala: PROARCA/CAPAS. < http://pdf.usaid.gov/pdf_docs/Pnacm456.pdf> (accessed march 3,2013)

Mendoza-Hernández M. 2015. Incremento diamétrico de cinco especies arbóreas con potencial maderable del bosque mesofilo de montaña en el centro de Verecruz. MSc. Thesis. Universidad Veracruzana, Mexico. <https:/www.uv.mx/mcef/ files/2018/04/Tesis.pdf $>$ (accessed September 25, 2018).

Muñiz-Castro M, Williams-Linera G, Benítez-Malvido J. 2015. Restoring montane cloud forest: establishment of three Fagaceae species in the old fields of central Veracruz, Mexico. Restoration Ecology 23: 26-33.

DOI: http://doi.org/10.1111/rec.12155.

Murcia C. 1997. Evaluation of Andean alder as a catalyst for recovery of tropical cloud forest in Colombia. Forestry Ecology and Management 99: 163-170.

DOI: http://doi.org/10.1016/S0378-1127(97)00202-8.

Orrego S, del Valle JI. 2001. Existencias y tasas de crecimiento neto de la biomasa y del carbono en bosques primarios y secundarios de Colombia. In: Simposio Internacional Medición y Monitoreo de la Captura de Carbono en Ecosistemas Forestales. Valdivia, Chile. <https://www.uach.cl/procarbono/ pdf/simposio_carbono/26_Orrego.PDF $>$ (accessed January 17, 2018).

Pareliussen I, Gunilla E, Olsson A, Armbruster WS. 2006. 
Factors limiting the survival of native tree seedlings used in conservation efforts at the edges of forest fragments in upland Madagascar. Restoration Ecology 14: 196-203. DOI: http://doi.org/10.1111/j.1526-100X.2006.00121.x.

Pompa-García M, Sigala-Rodríguez JA, Jurado E, Flores J. 2017. Tissue carbon concentration of 175 Mexican forest species. Forest - Biogeosciences and Forestry 10: 754-758. DOI http://doi.org/10.3832/ifor2421-010.

R Core Team. 2013. R: A language and environment for statistical computing. R Foundation for Statistical Computing, Vienna, Austria. <http://www.R-project.org/> (accessed january 13, 2013).

Ramírez-Bamonde ES, Sánchez-Velásquez LR, Andrade-Torres A. 2005. Seedling survival and growth of three species of Montane cloud forest in Mexico, under different canopy treatments. New Forests 30: 95-101. DOI: http://doi.org/10.1007/s11056-004-5397-5.

Rodríguez-Laguna R, Jiménez-Pérez J, Aguirre-Calderón OA, Treviño-Garza EJ. 2006. Estimación del carbono almacenado en un bosque de niebla en Tamaulipas, México. CienciaUANL 9: 179-187.

Russo RO. 1990. Evaluating Alnus acuminata as a component in agroforestry systems. Agroforestry Systems 10: 241-252. DOI: http://doi.org/10.1007/BF00122914.

Russo RO. 2005. Nitrogen Fixing Trees with Actinorhiza in Forestry and Agroforestry. In: Werner D, Newton WE, eds. Nitrogen Fixation in Agriculture, Forestry, Ecology, and the Environment. Netherlands: Springer. ISBN: 10 1-4020-3542-x.

Rzedowski J. 2006. Vegetación de México. 1ra. Edición digital, Comisión Nacional para el Conocimiento y uso de la Biodiversidad. México. <http://www.biodiversidad.gob.mx/publicaciones/librosDig/pdf/VegetacionMx_Cont.pdf $>$ (accessed January 21, 2018).

Sánchez-Velásquez LR, Ramírez-Bamonde E, Andrade-Torres A, Rodríguez-Torres P. 2008. Ecología, florística y restauración del bosque mesófilo de montaña. In: Sánchez-Velásquez LR, Galindo-González J, Díaz-Fleischer F, eds. Ecología, Manejo y conservación de los Ecosistemas de Montaña en México 10-32.Comisión Nacional para el Conocimiento y Uso de la Biodiversidad (CONABIO). Ciudad de México:
Universidad Veracruzana y Mundi Prensa. ISBN 978-9687462-57-8.

Santiago LS, Wright SJ. 2007. Leaf functional traits of tropical forest plants in relation to growth form. Functional Ecology 21: 19-27.

DOI: http://doi.org/10.1111/j.1365-2435.2006.01218.x.

Shimamoto CY, Botosso PC, Marques MC. 2014. How much carbon is sequestered during the restoration of tropical forests? Estimates from tree species in the Brazilian Atlantic forest. Forest Ecology and Management 329: 1-9. DOI: http://doi.org/10.1016/j.foreco.2014.06.002.

Siqueira JO, Saggin OJ. 2001. Dependency on arbuscular mycorrhizal fungi and responsiveness of some Brazilian native woody species. Mycorrhiza 11: 245-255. DOI: http://doi.org/10.1007/s005720100129.

Spracklen DV, Righelato R. 2014. Tropical montane forests are a larger than expected global carbon store. Biogeosciences 11: 2741-2754. DOI: http://doi.org/10.5194/bg-11-2741-2014.

Spracklen DV, Righelato R. 2016. Carbon storage and sequestration of re-growing montane forests in southern Ecuador. Forest Ecology and Management 364: 139-144. DOI: http://doi.org/10.1016/j.foreco.2016.01.001.

Swamy SL, Mishra A. 2014. Comparison of biomass and C storage in three promising fast growing tree plantations under agroforestry system in sub-humid tropics of Chhattisgarh. Universal Journal of Agricultural Research 2: 284-296. DOI: http://doi.org/10.13189/ujar.2014.020802

Vázquez-Yanes C. 1998. Trema micrantha (L.) Blume (U1maceae): a promising neotropical tree for site amelioration of deforested land. Agroforestry Systems 40: 97-104. DOI: http://doi.org/10.1023/A:1006063010677.

Velázquez E, Gómez-Sal A. 2009. Different growth strategies in the tropical pioneer tree Trema micrantha during succession on a large landslide on Casita Volcano, Nicaragua. Journal of Tropical Ecology 25: 249-260. DOI: http://doi.org/10.1017/S0266467409006026.

Yoda K, Kira T, Ogawa H, Hozumi K. 1963. Intraspecific competition among higher plants. XI. Self-thinning in overcrowded pure stands under cultivated and natural conditions. Journal of Biology of Osaka City University 14: 107-129.

Editor de sección: Pedro Valverde

Contribución de autores: MLAY: Participó en campo y manuscrito. LRSV: En diseño, campo, análisis, redacción y financiamiento. DMR: Participó en campo y redacción. YP: En diseño, financiamiento y manuscrito. SPIZ: En análisis de datos y manuscrito. EA: Diseño y manuscrito. MRPL: Coordinó, participó en campo, análisis, redacción y financiamiento. 